\title{
Heat Transfer in Turbulent Shear Flow
}

\author{
W. D. RANNIE* \\ California Institute of Technology
}

\section{SUMMARY}

The problems of heat transfer in turbulent shear flow along a smooth wall are discussed from the point of view of von Kármán's well-known 1939 paper on the analogy between fluid friction and heat transfer. Methods for extending the analysis to higher Prandtl Numbers are suggested.

\section{SyMBOLS}

$B=$ constant in turbulent core velocity profile

$C_{f}=2 \tau_{0} / \rho u_{m}^{2}=$ friction coefficient

$C_{h}=q_{0} / \rho c_{p} U_{m}\left(T_{w}-T_{m}\right)=$ heat-transfer coefficient

$c_{p}=$ specific heat at constant pressure

$D=$ pipe diameter

$k=$ thermal conductivity

$K=$ constant in turbulent core velocity profile

$K_{1}=$ constant in wall layer profile

$N u=q_{0} D /\left(T_{w}-T_{m}\right) k=$ Nusselt number

$q=$ heat-transfer rate per unit area in $y$-direction, $q_{0}$ the value at the wall

$R e=D u_{m} / \nu=$ Reynolds Number

$T=$ local mean temperature, $T_{w}$ at the wall, $T_{m}$ pipe mean

$u=$ local mean velocity along wall, $u_{m}$ pipe mean

$u_{\tau}=\sqrt{r_{0} / \rho}=$ friction velocity

$y=$ coordinate distance from wall

$y^{*}=y u_{\tau} / \nu=$ dimensionless distance from wall, $y_{1}{ }^{*}$ to edge of turbulent core, $y_{2}{ }^{*}$ to outermost distance of appreciable molecular transport of heat or momentum.

$\epsilon=$ eddy viscosity

$\kappa \quad=k / \rho c_{p}=$ thermal diffusivity

$\mu \quad=$ viscosity

$\nu \quad=\mu / \rho=$ kinematic viscosity

$\rho=$ fluid density

$\sigma=\mu c_{p} / k=$ Prandt 1 Number

$\tau=$ shearing stress, $\tau_{0}$ at the wall

\section{INTRODUCTION}

T HE FIRST IMPORTANT STEP in the understanding of heat transfer in turbulent flow was made by Reynolds ${ }^{1}$ in 1874 when he postulated the analogy between heat and momentum transfer in turbulent shear flow. For turbulent shear flow parallel to the $x$-axis, with velocity and temperature gradients predominant in the $y$-direction, the analogy can be expressed as

$$
\frac{-q}{\rho C_{p} d T / d y}=\frac{\tau}{\rho d u / d y}
$$

where $q$ is the rate of heat transfer per unit area in the $y$-direction, and $\tau$ is the shearing stress. For laminar shear flow, $q=-k d T / d y$ and $\tau=\mu d u / d y$, and one sees that the expression (1) above holds if, and only if, the Prandtl Number $\sigma$ is unity. Hence the Reynolds analogy is strictly correct for laminar shear flow provided the fluid properties are such that $\sigma=1$. The

* Robert H. Goddard Professor, Daniel and Florence Guggenheim Jet Propulsion Center.
Prandtl Numbers of most gases are close to unity ( $\sigma \cong 0.75$ ), so the Reynolds analogy is approximately correct up to the wall even though the flow is laminar close to the wall.

The first extensions of the application of Reynolds analogy to fluids with Prandtl Numbers differing from unity were made by Taylor ${ }^{2}$ in 1919 and Prandt $^{3}$ in 1926. The extensions consisted essentially of dividing the flow into two layers: a laminar layer next to the wall where the transport processes were assumed entirely molecular, and a turbulent region beyond where the molecular transport processes were neglected. A1though these methods gave an improvement over the application of the Reynolds postulate for Prandtl Numbers differing moderately from unity, they suffered from the rather arbitrary choice of the thickness of the laminar layer.

In 1934, von Kármán ${ }^{4}$ made a suggestion for improving the heat-transfer theory of fluids with Prandtl Numbers differing from unity, and, in 1939, he carried out his own suggestion in detail. This second paper, "The Analogy Between Fluid Friction and Heat Transfer," was published in the Transactions of the American Society of Mechanical Engineers and has become a classic to those working in the field of heat transfer. The significant step made by von Kármán was to introduce a buffer layer between a laminar sublayer next to the wall and the turbulent core. In the thin laminar sublayer, the transport processes were assumed purely molecular; in the turbulent core, the Reynolds analogy was used; while in the buffer layer, both molecular and turbulent transports were assumed, the first decreasing and the second increasing with distance from the wall. The Reynolds analogy was applied to the turbulent components of the transport terms within the buffer layer.

The importance of von Kármán's contribution consisted largely of the very clear demonstration of the essential transport phenomena. He confirmed the results of his analysis by comparison with heat-transfer measurements for water. The particular choice of laminar sublayer thickness was intended for a range of Prandtl Numbers moderately larger than unity. It is rather surprising that the concepts introduced by von Kármán have had, since that time, so little extension to liquids with higher Prandtl Numbers. Some of the problems in such extension are discussed below.

\section{Momentum and Energy Transfer}

Consider plane shear flow or the flow in a pipe far from the center; the momentum and energy equations, 
averaged over the turbulent fluctuations, become

$$
\begin{aligned}
\tau_{0} & =-\rho \overline{u^{\prime} v^{\prime}}+\mu(d u / d y) \\
-q_{0} & =-\rho c_{p} \overline{T^{\prime} v^{\prime}}+k(d T / d y)
\end{aligned}
$$

The total shear stress $\tau_{0}$, assumed constant, is the sum of the Reynolds stress $-\rho \overline{u^{\prime} v^{\prime}}$ and the laminar stress $\mu(d u / d y)$; and the rate of heat transfer $q_{0}$, also assumed constant, is the sum of analogous components. It is convenient to introduce an eddy viscosity $\epsilon$ and to write Eq. (2) as

$$
\tau_{0} / \rho=(\epsilon+\nu)(d u / d y)
$$

where $\epsilon$ is the turbulent counterpart of the kinematic viscosity $\nu$. The latter is, of course, a property of the fluid, whereas $\epsilon$ varies with $y$ and must vanish at $y=0$ for a smooth wall. The energy transport equation may be written in a similar form

$$
-\left(q_{0} / \rho c_{p}\right)=\left(\epsilon_{T}+\kappa\right)(d T / d y)
$$

where $\epsilon_{T}$ is the eddy diffusivity.

Reynolds analogy is equivalent to the assumption that $\epsilon_{T}=\epsilon$ for flows where the molecular components are negligible. Following von Kármán, the same assumption will be used even when $\nu$ and $\kappa$ are not negligible compared with $\epsilon$. The Reynolds analogy does not hold in all types of shear flow. It is well known that the turbulent temperature wake behind a heated body spreads faster than the velocity wake; for instance, Corrsin $^{5}$ has found a "turbulent Prandtl Number" $\epsilon / \epsilon_{T} \cong 0.75$ in a particular example. This is to be expected in a jet or wake far downstream where the $u^{\prime}$ and $v^{\prime}$ velocity components are of comparable magnitude, because the momentum transport involves transport of a component of a vector and energy involves the transport of a scalar. Close to a wall the flow is confined strongly to planes parallel to the wall, and it seems likely that the transport of the $u$ component of momentum is similar to transport of a scalar quantity. Measurements of heat transfer in pipes for gases, where the Prandtl Number correction is small, support the Reynolds analogy for shear flow near a wall.

Returning to Eqs. (4) and (5) and introducing the friction velocity $u_{\tau}=\sqrt{\tau_{0} / \rho}$ and a dimensionless distance from the wall $y^{*}=y u_{\tau} / \nu$, the equations can be rewritten as

$$
\begin{aligned}
\frac{d\left(u / u_{\tau}\right)}{d y^{*}} & =\frac{1}{(\epsilon / \nu)+1} \\
-\frac{\rho c_{p} u_{\tau}}{q_{0}} \frac{d T}{d y^{*}} & =\frac{1}{(\epsilon / \nu)+(1 / \sigma)}
\end{aligned}
$$

These equations can be integrated in principle to give the velocity and temperature profiles; these are

$$
\begin{gathered}
\frac{u}{u_{\tau}}=\int_{0}^{y_{2} *} \frac{d y^{*}}{(\epsilon / \nu)+1}+\int_{y_{2}^{*}}^{y^{*}} \frac{d y^{*}}{\epsilon / \nu} \\
\frac{\rho C_{p} u_{\tau}}{q_{0}}\left(T_{r}-T\right)=\int_{0}^{y_{2} *} \frac{d y^{*}}{(\epsilon / \nu)+(1 / \sigma)}+\int_{y_{2} *}^{y^{*}} \frac{d y^{*}}{\epsilon / \nu}
\end{gathered}
$$

where $y_{2}{ }^{*}$ is sufficiently large that 1 or $1 / \sigma$, whichever is larger, becomes negligible compared with $\epsilon / \nu$. If $\sigma \ll 1$, the formulation above may not be satisfactory, and modifications similar to those used by Martinelli ${ }^{6}$ can be introduced.

The upper limit $y^{*}$ for the second integral in the expression for $u / u_{\tau}$ can always be chosen so $u=u_{m}$, the average velocity, as for a pipe. Then, with a small error, the temperature evaluated at the same $y^{*}$ is the average fluid temperature $T_{m}$ (see Appendix for a more accurate evaluation). With these limits for the integrals, the equations above are subtracted to give

$$
\left(\rho c_{p} u_{\tau} / g_{0}\right)\left(T_{w}-T_{m}\right)-\left(u_{m} / u_{\tau}\right)=F(\sigma)
$$

where $F(\sigma)=\int_{0}^{y_{2} *}\left[\frac{1}{(\epsilon / \nu)+(1 / \sigma)}-\frac{1}{(\epsilon / \nu)+1}\right] d y^{*}$

The friction coefficient $C_{f}$ and the heat-transfer coefficient (Stanton number) $C_{h}$ are defined by the relations

$$
\begin{aligned}
C_{f} / 2 & =\tau_{0} /\left(\rho u_{m}^{2}\right) \\
C_{h} & =g_{0} /\left[\rho c_{p} u_{m}\left(T_{w}-T_{m}\right)\right]
\end{aligned}
$$

Multiplying Eq. (6) through by $u_{m} / u_{\tau}$, the equation relating heat transfer and friction is

$$
1 / C_{h}=\left(2 / C_{f}\right)+F(\sigma) \sqrt{2 / C_{f}}
$$

where $F(\sigma)$ is given by Eq. (7) above. For $\sigma=1$, $F(\sigma)=0$, and the Reynolds analogy holds in both laminar and turbulent regions giving $C_{h}=C_{f} / 2$.

\section{The Distribution of Eddy Viscosity}

For Prandtl Numbers differing from unity, the relation between the heat-transfer and friction coefficients depends on the distribution of eddy viscosity $\epsilon$ between $y^{*}=0$ and $y^{*}=y_{2}{ }^{*}$. The most direct way of determining $\epsilon$ is from the velocity profile since

$$
\frac{\epsilon}{\nu}=\frac{1}{d\left(u / u_{\tau}\right) / d y^{*}}-1
$$

For $y^{*}>30$-i.e., in the fully turbulent region-the von Kármán logarithmic formula can be used; this was given in the form

$$
u / u_{\tau}=(1 / \sqrt{K}) \ln y^{*}+B
$$

and the constants $1 / \sqrt{ } \bar{K} \cong 2.5, B=5.5$ are well established by experiments on pipes. Hence

$$
\epsilon / \nu=\sqrt{K} y^{*}-1 \quad y^{*}>30
$$

For the range $0<y^{*}<30$, von Kármán approximated the measured velocity profile by $u / u_{\tau}=y^{*}$ in the laminar sublayer $0<y^{*}<5$, and by a relation of the form of Eq. (9), with different constants, in the buffer layer $5<y^{*}<30$. Then $\epsilon / \nu=0$ for $y^{*}<5$, and this is a satisfactory approximation for moderately large Prandtl Numbers.

There is no reason for believing that $\epsilon / \nu$ should vanish completely except at the wall itself. The velocity profile near the wall would be virtually unaffected by 
an error in the very small value of $\epsilon / \nu$ in that region. However, an examination of Eq. (7) shows that for $\sigma \gg 1$, the very small values of $\epsilon / \nu$ close to the wall can have a profound influence on the value of the first integral. There is no possibility of determining $\epsilon / \nu$ accurately for $y^{*}<5$ from measurements because the velocity gradient is required, and it is impractical to attempt to find these from the measured profile. Even the remarkably good experiments of Reichardt, ${ }^{7}$ Laufer, ${ }^{8}$ and Skinner ${ }^{9}$ are of no help in this regard.

Since it does not seem possible to find heat-transfer coefficients for high Prandtl Numbers from measured velocity profiles, it is natural to examine the possibility of finding the velocity profile from heat-transfer measurements. In principle, this is attractive because temperature differences, flow rates, and pressure drop can be measured quite accurately. By heat-transfer measurements on fluids of different Prandtl Numbers, $F(\sigma)$ could be determined as a function of $\sigma$, and Eq. (7) then is an integral equation for $\epsilon / \nu$. The difficulty with heat-transfer measurements in the past has been that the physical properties, particularly the viscosity, are sensitive functions of temperature for nonmetallic liquids. In order to have an appreciable amount of heat transferred from the wall, it is necessary to use a large temperature difference between the wall and the bulk of the fluid. Frequently, the viscosity changes by a factor of two or three or more from the wall to the edge of the turbulent core. Some attempt is usually made to allow for the viscosity variation by various averaging processes. Reported heat-transfer measurements often show "scatter" of \pm 20 per cent; it seems likely that the spread is not so much an indication of experimental error as a gross oversimplification of the influence of variation of viscosity. Careful experiments of the type made by Eagle and Ferguson ${ }^{10}$ on water, with extrapolation to zero temperature differences, are needed for liquids with higher Prandtl Numbers.

\section{Proposed Velocity Profile}

In the absence of sufficiently precise heat-transfer measurements, the writer attempted to find a reasonable velocity profile for the region close to the wall, so the eddy viscosity determined from the velocity led to satisfactory variation of heat transfer with Prandtl

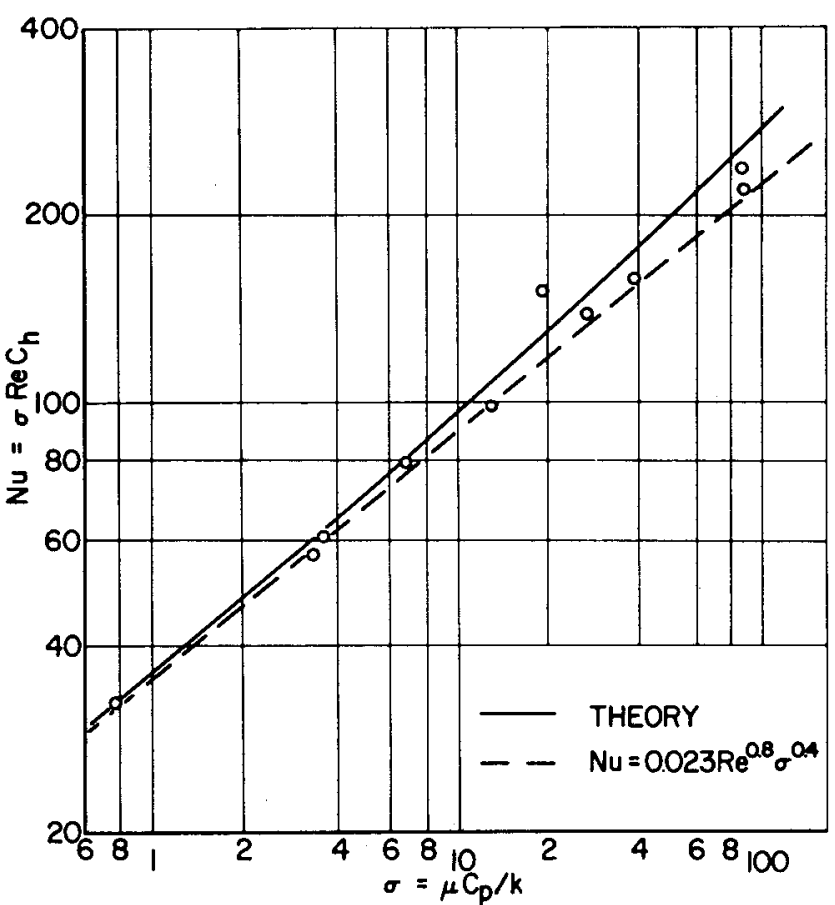

FIg. 1. Dependence of Nusselt number on Prandtl Number at $R e=10^{4}$. (Experimental data from Sherwood.)

Number. The simplest analy tical expression that fitted the required conditions was of the form

$$
u / u_{\tau}=\left(1 / \sqrt{K_{1}}\right) \tanh \left(\sqrt{K_{1}} y^{*}\right)
$$

This profile was joined to the logarithmic profile, Eq. (9), at a distance $y_{1}{ }^{*}$, so the velocity and velocity gradient were continuous at the junction. The constant $K_{1}$ and the distance $y_{1}{ }^{*}$ were determined from these two conditions giving

$$
y_{1}^{*}=27.5 \quad 1 / \sqrt{K_{1}}=14.53
$$

Comparison of the velocity profile, Eq. (11), with experimental results gives quite satisfactory agreement; of course many other analytical forms for the profile would be equally satisfactory, but this one is very convenient.

With the velocity profile as given by Eq. (11), the corresponding eddy viscosity is

$$
\epsilon / \nu=\sinh ^{2}\left(\sqrt{K_{1}} y^{*}\right)
$$

and the function $F(\sigma)$ can be evaluated in closed form.

$$
\left.\begin{array}{rl}
F(\sigma) & =\frac{\sigma}{\sqrt{K_{1}} \sqrt{\sigma-1}} \tan ^{-1}\left(\sqrt{\sigma-1} \sqrt{K_{1} y_{1}}\right)-B-\frac{1}{\sqrt{K}} \ln \left(y_{1}^{*}-\frac{1}{2 \sqrt{K}}+\frac{1}{\sqrt{K} \sigma}\right) \quad \sigma>1 \\
& =\frac{\sigma}{\sqrt{K_{1}}} \frac{}{\sqrt{1-\sigma}} \tanh ^{-1}\left(\sqrt{1-\sigma} \sqrt{K_{1}} y_{1}^{*}\right)-B-\frac{1}{\sqrt{K}} \ln \left(y_{1}^{*}-\frac{1}{2 \sqrt{K}}+\frac{1}{\sqrt{K} \sigma}\right) \quad \sigma<1
\end{array}\right\}
$$

The asymptotic form for $F(\sigma)$ as $\sigma$ becomes large is

$$
F(\sigma) \rightarrow 22.8 \sqrt{\sigma}-29.1 \quad \text { as } \sigma \rightarrow \infty
$$

and this is only 5 per cent smaller than the correct value at $\sigma=10$.
In order to show that the value of $F(\sigma)$ is in agreement with experiment, a comparison is made for the Prandt1 Number range between 1 and 100 in Fig. 1. The experimental points are those of Sherwood as reported by McAdams. ${ }^{11}$ The friction coefficient was evaluated 
from the empirical relation $C_{f}=0.046 / R e^{0.2}$. As can be seen, the fit is reasonably good; it must be remembered that the variations in viscosity that occurred near the wall in the experiments are ignored. Also shown is one of the well-known empirical formulas ${ }^{12}$ for the range $1<\sigma<120$.

$$
C_{h}=0.023(R e)^{-0.2} \sigma^{-0.6}
$$

The theory above implies that $C_{h} \infty \sigma^{-0.5}$ as $\sigma$ becomes very large. Few reliable measurements of heat transfer at very high Prandtl Numbers $(\sigma>500)$ have been made, but those that are available ${ }^{13}$ seem to indicate that $C_{h} \infty \sigma^{-2 / 3}$ for $\sigma$ very large. This result, if correct, would require that $\epsilon / \nu \propto y^{* 3}$ for $y^{*}$ very small. According to Eq. (13), the turbulent and molecular transports of heat are equal at a value of $y^{*}$ given by $\sinh \sqrt{K_{1}} y_{0}^{*}=1 / \sqrt{\sigma}$; hence at $\sigma=100$ $y_{0}{ }^{*} \cong 1.45$. Then one would expect that the velocity profile, Eq. (11), describes the actual velocity reasonably well for $y^{*} \sim 1.5$ but not necessarily for smaller values of $y^{*}$, unless the agreement indicated in Fig. 1 can be confirmed at higher values of the Prandtl Number. Many other forms for the velocity profile that have been proposed - for instance that of Deissler ${ }^{14}$ are not consistent with heat-transfer measurements at the higher Prandt1 Numbers.

\section{Fluids with Variable Physical Properties}

As was mentioned previously, heat-transfer measurements in liquids with Prandtl Numbers larger than unity usually involve variable viscosity. If for no other reason than interpretation of experiments, an understanding of the influence of variable physical properties on friction and heat transfer is desirable. An important simplification resulting from high Prandtl Numbers is that most of the temperature difference occurs in the layer between the wall and $y^{*}=30$, so the turbulent core is essentially isothermal.

Measurements of Laufer ${ }^{8}$ and others of the magnitude of the velocity fluctuations near a smooth wall show that the maximum values occur at $y^{*} \cong 25$ or 30 and decrease rapidly to zero at the wall. It seems probable that the wall layer within these limits is inherently stable and that the velocity fluctuations are responses of a highly damped layer to disturbances arising outside. The fluctuations inside the wall layer would then have no direct influence on the outside flow.

If the temperature variation is confined to the damped layer of fluid next to the wall, the viscosity variation is confined to this region as well. The turbulent core is isothermal and can be considered as having known velocity gradient distribution and as producing known disturbances at the edge of the wall layer. The response at any point of the wall layer can then in principle be determined, even if the viscosity varies strongly through the layer. The turbulent shear stress $-\rho \overline{u^{\prime} v^{\prime}}$ will depend on the viscosity variation, but, with the model above, there is some hope of estimating this dependence. The writer ${ }^{15}$ has attempted to use such a model for the wall layer to determine $\epsilon / \nu$ as a function of the viscosity variation and hence to find the friction coefficient and the heat-transfer coefficient. Although the results are promising, confirmation will not be possible until more reliable heat-transfer and friction measurements are made for liquids at high Prandtl Numbers.

In gases, about half the temperature difference between the wall and the mean temperature occurs in the wall layer so that the turbulent core is not isothermal. Then the disturbances of the damped wall layer cannot be considered as known because the turbulent core cannot be identified with a similar one in isothermal flow. Further, the density fluctuations in gases may introduce other complications not found in liquids. An understanding, or even a reliable description of the turbulent transport processes close to a wall for high rates of heat transfer, seems much farther in the future for gases than for liquids at high Prandtl Numbers.

\section{APPENDIX}

A more satisfactory derivation of Eq. (8) can be carried out by defining mean velocity and mean temperature for pipe flow with appropriate weighting factors-e.g.,

$$
\begin{gathered}
\frac{u_{m}}{u_{\tau}}=\frac{2}{R^{* 2}} \int_{0}^{R^{*}} \frac{u}{u_{\tau}}\left(R^{*}-y^{*}\right) d y^{*} \\
T_{w}-T_{m}=\frac{2}{R^{* 2}\left(u_{m} / u_{\tau}\right)} \int_{0}^{R^{*}}\left(T_{w}-T\right) \frac{u}{u_{\tau}} \times \\
\left(R^{*}-y^{*}\right) d y^{*}
\end{gathered}
$$

where $R^{*}=R u_{r} / \nu$ and $R$ is the pipe radius. If the evaluation of the integrands is made with terms of order $1 / R^{*}$ and higher neglected, Eq. (8) is replaced by

$$
1 / C_{h}=\left(2 / C_{f}\right)+F(\sigma) \sqrt{2 / C_{f}}+5 / 4 K
$$

The additional term on the right, approximately 8.8 , is an unimportant correction for most applications.

\section{REFERENCES}

${ }^{1}$ Reynolds, O., Collected Papers, Vol. I, pp. 81-85.

2 Taylor, G. I., ARC R\&M No. 272, 1919.

a Prandtl, L., Physik. Zeitsch. Vol. 29, pp. 487-489, 1928.

${ }^{4}$ von Kármán, Th., Some Aspects of the Turbulence Problem, Proc. 4th Int. Cong. for App. Mech., pp. 54-91, 1934; The Analogy between Fluid Friction and Heat Transfer, Trans. ASME Vol. 61, pp. 705-710, 1939.

${ }^{5}$ Corrsin, S., Investigation of Flow in an Axially Symmetrical Heated Jet of A ir, NACA Wartime Report W-94, December, 1943.

${ }^{6}$ Martinelli, R. C., Heat Transfer in Molten Metals, Trans. ASME Vol. 63, pp. 447-455, 1941.

' Reichardt, H., Die Warmeubertragung un turbulenten Reibungschichten, ZAAM, Vol. 20, pp. 297-328, 1940.

${ }^{8}$ Laufer, J., Some Recent Measurements in a Two-Dimensional Turbulent Channel, Journal of the Aeronautical Sciences, Vol. 17, No. 5, pp. 277-287, May, 1950.

${ }^{9}$ Skinner, G. T., Mean Speed Measurements in Turbulent Channel Flow, Thesis, California Institute of Technology, 1951. 
${ }^{10}$ Eagle, A., and Ferguson, R. M., On the Coefficient of Heat Transfer From the Internal Surface of Tube Walls, Proc. Roy. Soc. London A, Vol. 127, p. 540, 1930.

11 McAdams, W. H., Heat Transmission, 2nd Ed., p. 168; McGraw-Hill Book Company, Inc., New York, 1942.

12 McAdams, W. H., Heat Transmission, 3rd Ed., p. 219; McGraw-Hill Book Company Inc., New York, 1954.
${ }^{13}$ Murphree, E. V., Relation Between Heat Transfer and Fluid Friction, Industrial and Engineering Chemistry, Vol. 24, pp. 726-736, 1932.

${ }_{14}$ Deissler, R. G., Analytical Investigation of Turbulent Flow in Smooth Tubes with Variable Fluid Properties for Prandtl Number of 1, NACA TN 2242, December, 1950.

ts Rannie, W. D., Thesis, California Institute of Tech., 1951. 